\title{
Premenstrual syndrome: an update on definitions, diagnosis and management
}

\author{
Moira Connolly
}

Most articles on the management of the premenstrual syndrome (PMS) begin by noting that the term "premenstrual tension" was first coined by R. T. Frank in 1931 and "pre-menstrual syndrome" by Greene \& Dalton in 1953. However, in 1847, Dr Ernst F. von Feuchtersleben wrote:

"The menses in sensitive women is almost always attended by mental uneasiness, irritability and sadness." (quoted by Rubinow \& Schmidt, 1995)

Difficulties remain in defining the condition, identifying its aetiology and in understanding why some women are 'sensitive' to developing this cyclical condition, which is linked to the menstrual cycle and characterised by mainly affective symptoms such as irritability and dysphoria.

In this paper, we aim to summarise what we currently understand about PMS and the more narrow and operationally defined 'premenstrual dysphoric disorder' (PMDD; American Psychiatric Association (APA), 1994), which probably equates with the most severe end of the PMS spectrum. In addition, the assessment and management of patients will be described with reference to both the physical and psychological components of severe PMS.

\section{Definition and diagnosis of premenstrual syndrome}

Premenstrual syndrome(s) has (have) been variously defined. The National Institute of Mental Health definition states that premenstrual changes or:

\begin{abstract}
"the cyclic occurrence of symptoms that are of sufficient severity to interfere with some aspects of life and which appear with consistent and predictable relationships to menses" (Endicott et al, 1981)
\end{abstract}

show at least a $30 \%$ increase from the intensity of symptoms measured in the follicular phase, that is, in days 5-10 of the menstrual cycle, compared with those measured in the premenstrual phase (on the 6 days before menstruation). In addition, premenstrual changes must be prospectively documented for at least two consecutive menstrual cycles. Some research authorities stipulate a $50 \%$ worsening of symptoms by the premenstrual phase (Steiner \& Wilkins, 1996). The 30\% change in ratings of symptoms has been shown to be too liberal and a poor discriminator when comparing women with self-reported severe PMS, women using contraceptives whose natural cyclicity has been suppressed and women with normal cyclicity who report no premenstrual symptoms (Gallant et al, 1992). Gallant et al emphasise that what may be more clinically relevant is women's perceptions of variations in social and occupational functioning and "the way in which having PMS is meaningful in a woman's life". The authors' concern is that ever more stringent criteria might result in the exclusion from studies or treatment of significantly troubled individuals.

The diagnosis of PMS was operationalised with the introduction of the term 'late luteal phase dysphoric disorder' (LLPDD) into DSM-III-R (APA, 1987) under the section headed 'Proposed diagnostic categories needing further study'. Subsequently, a work group on LLPDD reported to the DSM-IV Task Force, culminating in its inclusion in DSM-IV

Moira Connolly is a consultant general psychiatrist at Gartnavel Royal Hospital (Glasgow G12 0XH) with interests in psychiatric rehabilitation and cognitive-behavioural therapy (CBT). She is a supervisor on the South of Scotland CBT course and honorary senior lecturer in the Department of Psychological Medicine, University of Glasgow. 
(APA, 1994) as 'premenstrual dysphoric disorder' in the section 'Mood disorders not otherwise specified', with its clinical criteria laid out in Appendix B - 'For further study' (see Box 1).

It is widely agreed that the dysphoric symptoms of PMS are among the most troublesome. This is reflected in the DSM-IV diagnostic criteria, with 10 of 11 symptoms being emotional or behavioural in nature, while the 11th covers multiple physical symptoms. In ICD-10 (World Health Organisation (WHO), 1992), it is listed as a physical disorder N94-3, 'premenstrual tension syndrome', under 'Pain and other conditions associated with female genital organs and the menstrual cycle'. There is no requirement for a minimum number of symptoms or for functional impairment to make the ICD-10 diagnosis of PMS. For the purposes of this article, the abbreviation PMS will be used, as few studies have exclusively dealt with patient populations whose diagnosis conforms to the narrower DSMIV definition.

DSM-IV allows the diagnosis of premenstrual dysphoric disorder to be made alongside other disorders, but offers no guidance on differentiating between the two. Two disorders may coexist or indeed be intricately related to one another. For example, persistent symptoms across the early follicular phase may represent a reaction to severe PMS. Alternatively, PMS may prove the final stressor leading to an inability to cope for someone with an underlying affective disorder. This may explain the increase in psychiatric hospital admission rates for women in weeks 1 and 4 of their menstrual cycle, reported by O'Dwyer et al (1997).

The association between dysphoric PMS and other physical and psychiatric disorders in womens'

Box 1 Research criteria for premenstrual dysphoric disorder (from DSM-IV, with kind permission from the American Psychiatric Association)

A. In most menstrual cycles during the past year, five (or more) of the following symptoms were present for most of the time during the last week of the luteal phase, began to remit within a few days after the onset of the follicular phase, and were absent in the week post-menses, with at least one of the symptoms being either (1), (2), (3), or (4):

1. markedly depressed mood, feelings of hopelessness, or self-depreciating thoughts

2. marked anxiety, tension, feeling of being 'keyed up' or 'on edge'

3. marked affective lability (e.g. feeling suddenly sad or tearful or increased sensitivity to rejection)

4. persistent and marked anger or irritability or increased interpersonal conflicts

5. subjective sense of difficulty in concentrating

6. lethargy, easy fatigability, or marked lack of energy

7. marked change in appetite, overeating, or specific food cravings

8. hypersomnia or insomnia

9. a subjective sense of being overwhelmed or out of control

10. other physical symptoms, such as breast tenderness or swelling, headaches, joint or muscle pain, a sensation of 'bloating', weight gain.

Note: In menstruating females, the luteal phase corresponds to the period between ovulation and the onset of menses, and the follicular phase begins with menses. In non-menstruating females (e.g. those who have had a hysterectomy), the timing of luteal and follicular phase may require measurement of circulating reproductive hormones.

B. The disturbance markedly interferes with work or school or with usual social activities and relationships with others (e.g. avoidance of social activities, decreased productivity and efficiency at work or school).

C. The disturbance is not merely an exacerbation of the symptoms of another disorder, such as major depressive disorder, panic disorder, dysthymic disorder, or a personality disorder (although it may be superimposed on any of these disorders).

D. Criteria A, B and C must be confirmed by prospective daily ratings during at least two consecutive symptomatic cycles. (The diagnosis may be made provisionally prior to this confirmation.) 
life cycles has been comprehensively reviewed by Halbreight (1996). These include other hormonally related disorders such as post-partum depression, dysphoric adverse effects with some contraceptive medication and dysphoric mood during sequential hormonal replacement therapy, in addition to major depressive disorders and seasonal affective disorder. Widening the net, a high lifetime comorbidity of dysphoric PMS has also been reported with dysthymic disorder, panic disorder, various anxiety disorders, alcohol and drug misuse, acute or chronic depression, various personality disorders and physical disorders such as hypertension, systemic lupus erythematosis and endometriosis.

The differential diagnosis of women presenting with premenstrual symptoms includes a broad range of psychiatric and physical disorders that can mimic, exacerbate, or coincide with PMS. It is imperative that history-taking and examination are detailed (Pearlstein \& Stone, 1998).

\section{Steps in assessment and diagnosis}

The literature on PMS is fraught with variations in diagnostic procedures, assessment schedules and outcome measures, making it difficult to make meaningful assessments of the efficacy of specific treatments. For example, Budeiri et al (1994) have reviewed the entry criteria and scales used for measuring treatment outcomes in 350 clinical trials of 115 drug treatments for PMS and recorded 65 different assessment scales. Useful recent reviews of clinically relevant diagnostic procedures and prospective rating scales are those of Steiner \& Wilkins (1996) and Pearlstein \& Stone (1998).

Symptoms must be rated prospectively in order to avoid the inaccuracies inherent in retrospective rating. These include incorrect symptom timing and amplification of symptom severity. Symptoms recorded over several cycles will illustrate any intercycle variability in the nature and severity of symptoms. Psychological stressors in the lives of individuals will vary irrespective of the cycle graphically illustrated in one study when an unpredicted symptom peak occurred with several women and the actual calendar date (as opposed to menstrual date) was noted to be 24 December (Sampson, 1988)!

Budeiri et al (1994) advocate using existing scales such as the Menstrual Distress Questionnaire (MDQ - 47 items), the Premenstrual Experience Assessment (PEA - 88 items) and the Premenstrual Assessment Form (PAF - 95 items), particularly when assessing treatment efficacy in a research setting. Shortened versions of these are in existence, for example, the Premenstrual Tension Scale (PMTS) derived from the MDQ, and although less fully evaluated are probably more acceptable to women prepared to keep prospective diaries. Other commonly used rating scales include the Daily Record of Severity of Problems (incorporating DSM-IV PMDD symptoms) and the Prospective Record of the Impact and Severity of Menstruation (PRISM).

Single sheets in calendar, visual analogue or diary forms, such as the Calendar of Premenstrual Experiences (COPE), are probably most acceptable in clinical practice. Other diagnostic instruments such as commonly used anxiety and depression questionnaires may also play a valid role in helping clinicians to assess symptom severity and identify comorbid disorders. For full details and references to specific scales, see Budeiri et al (1994) and Pearlstein \& Stone (1998).

Ideally, prospective daily ratings should demonstrate a premenstrual increase in symptom severity measurable as a percentage change, or in relation to an absolute severity requirement. This is achieved by dividing the cycle into phases and comparing the symptom scores for each phase. Despite limited agreement in the research literature as to phase identification, comparing average scores in the troublesome days premenstrually with those obtained in the days after menstruation would have most relevance clinically. Confirming subjectively reported functional impairment can be problematic, but the use of social adjustment scales and quality of life measures holds promise (see Box 2 for a summary of the assessment process).

Steiner \& Wilkins (1996) propose using both ICD10 and DSM-IV when assessing individual patients. Doing so helps to delineate the severity of symptoms, takes into account other possible comorbid conditions and helps guide appropriate treatment. They

Box 2 Summary of steps in the assessment process

Full history, relevant physical examination and investigation

Exclude the presence of any other physical or psychiatric illness

Prospective rating of symptoms using agreed questionnaires or calendar sheets for at least two cycles

Check for symptom patterns and particularly the presence of an increase in symptoms in the premenstrual phase 
recommend the use of the term 'premenstrual magnification', PMM, where other conditions such as depression are exacerbated in the premenstrual phase. Box 3 outlines the diagnostic categories that they propose for use in clinical practice.

\section{Demographics and aetiology}

Most women can detect the physiological changes associated with the pre-menstruum, but only between $3 \%$ and $8 \%$ experience severe symptoms in keeping with a diagnosis of PMDD (Steiner \& Wilkins, 1996), with the vulnerable years being the late 20s and early 30s (Freeman, 1995). Retrospective reporting and postal surveys account for some of the variations in prevalence studies.

\section{Gonadal steroids}

The link to the menstrual cycle strongly implicates gonadal sex steroids in the genesis of symptoms, however, endocrinological studies have not revealed convincing abnormalities to date. Basal and dynamic gonadal hormone studies have failed to show any luteal phase-specific biological abnormalities in PMS (Roca et al, 1996). A frequently cited study by Schmidt et al (1991) used a progesterone antagonist to manipulate PMS patients' hormones such that some were fooled as to where they were in their cycle by artificially inducing menstruation early. They then experienced PMS symptoms in what was hormonally changed to the early follicular phase, that is, immediately postmenstruation. This study suggests that hormonal events before the late luteal phase could set in motion interactions resulting in symptoms premenstrually and, from the psychological perspective, it also suggests that premenstrual symptoms cannot be explained solely by expectation bias.

\section{The role of serotonin}

Rapkin (1992) has summarised the role of serotonin in human and animal behaviour and the relevance of this research for the premenstrual syndrome. She cites studies on vervet monkeys, which demonstrated that the higher serotonin level seen in dominant males is not a fixed trait marker but is influenced by the social interactions the animal experiences. Subsequent alterations in whole blood serotonin levels become evident 7-10 days after changes to the animal's social experience. Rapkin suggests that women who do not have PMS may be offsetting the physiological changes triggered by ovulation and the ensuing tendency towards behavioural isolation that occur post-ovulation, by directly engaging in rewarding social interactions with other people. It is these rewarding interactions

Box 3 Useful clinical diagnostic categories (Steiner \& Wilkins, 1996)

Premenstrual syndrome (PMS)

Meet ICD-10 criteria for PMS but not DSM-IV PMDD. Criteria include mild psychological symptoms, bloating, weight gain, breast tenderness, swelling, aches and pains, poor concentration, sleep disturbance and appetite change. Only one of these symptoms is required but must be restricted to the luteal phase of the cycle, ceasing around menstruation.

Premenstrual dysphoric disorder (PMDD)

Meet DSM-IV criteria for PMDD (see Box 1). They have no other concurrent psychiatric disorder but may have a past history of psychiatric illness. Symptoms have occurred with most cycles in the past year and have interfered with social or occupational roles. Premenstrual worsening of symptoms is clearly evident and a $50 \%$ worsening by the luteal phase warrants pharmacological treatment.

Premenstrual magnification (PMM)

Women who receive this diagnosis may meet criteria for PMS or PMDD but also have a current major psychiatric disorder or an unstable medical condition.

Other psychiatric diagnosis only

Symptoms, although significant, show no relationship to the menstrual cycle.

No diagnosis

Symptoms, although disruptive, are not severe enough to warrant a diagnosis. 
that may lead to physiological changes resulting in increased serotonin levels and the maintenance of a stable mood. There may be several reasons why women with PMS may not engage in such rewarding behaviours in the latter half of their menstrual cycle, but environmental and relationship factors are probably important. And not engaging in rewarding social interactions may increase the probability of physiological changes in the direction of lowered serotonin levels and further troublesome psychological symptoms in women with PMS.

Carbohydrate craving is experienced by many women in the premenstrual week and sometimes throughout the cycle. In PMS, carbohydrate craving has been viewed as an attempt at self-medicating, with increased brain serotonin synthesis reported following carbohydrate intake (Wurtman, 1989).

With respect to the influence of gonadal hormones on serotonergic function, most information has come from animal studies and is not always consistent or conclusive. However, in general, decreased levels of gonadal hormones are associated with decreased serotonergic activity. In addition, there appears to be low activity of some serotonergic functions throughout the cycle and others in the luteal phase only in women with PMS compared with controls. This supports the assumption that women with PMS may have vulnerability traits that predispose to the development of dysphoric PMS, but also supports the view that some abnormalities of serotonergic function are state-related (reviewed by Halbreight, 1996). Further evidence for the involvement of serotonergic systems in dysphoric PMS comes from research studies that show a positive response to treatment with serotonin reuptake inhibitors (Eriksson, 1999).

\section{Other biological substrates}

Other biological substrates have been shown to be different in PMS patients compared with controls, but the differences exist throughout the menstrual cycle and therefore cannot solely explain symptoms confined to the luteal phase. These have included increased prevalence of abnormal thyroid stimulating hormone in response to thyrotropin-releasing hormone, decreased slow-wave sleep, altered melatonin secretion, deceased red blood cell magnesium, blunted growth hormone and cortisol responses to L-tryptophan, and increased cortisol response to corticotrophin-releasing hormone infusion. Decreases in plasma $\beta$-endorphin have also been noted, although not consistently (Rubinow, 1992).

What is certain is that normal cyclical ovarian function is central to the disorder and abolishing it by either medical or surgical oophorectomy can prove curative. It is generally agreed that there is no single biological substrate that can be considered causal in isolation, but a bio-psychosocial model probably best fits the clinical picture and should underpin approaches to treatment.

\section{Pharmacological treatments for PMS}

Halbreight (1996) divides pharmacological treatments into two categories:

(a) symptomatic treatment; and

(b) ovulation suppression.

\section{Symptomatic treatment}

By far the biggest advance in symptomatic treatment for PMS in recent years has been the use of selective serotonin reuptake inhibitors (SSRIs). Most serotonergic-enhancing antidepressants have been shown to be effective in the treatment of PMS in comparison with placebo, for example, fluoxetine, citalopram, paroxetine, sertraline and clomipramine. Nonserotonergic antidepressants such as maprotiline and desipramine have been less helpful in comparison with SSRIs. Recent studies have shown that some SSRIs given in the latter half of the cycle can be as effective as continuous daily dosing. In the case of citalopram, half-cycle dosing was better than continuous dosing - an unexpected finding that possibly suggests that intermittent SSRI use may avoid the development of tolerance (Eriksson, 1999).

It has been postulated that some women with PMS may have decreased serotonergic activity across the menstrual cycle as a trait, and during the luteal phase further abnormalities of some serotonergic functions may occur on a state-related basis. This may underpin the view that using an SSRI in the luteal phase only, or increasing the dose at this time, is a treatment rationale with some promise. The beneficial effects of SSRIs on dysphoric symptoms are evident soon after the initiation of treatment, suggesting that the mode of action may be different to that in other depressive illnesses, where symptomatic improvements can take several weeks to emerge. Other serotonergic agents have been shown to have variable promise in the treatment of dysphoric PMS, but not all have been subjected to placebo-controlled trials. These include nefazodone, the serotonin agonist D-fenfluramine, the serotonin 1a-receptor agonist buspirone and L-tryptophan. 
Many miscellaneous treatments have been used with variable results. Alprazolam, a benzodiazepine with antidepressant and anxiolytic properties, has been subjected to controlled trials of use in the luteal phase with mixed results. It is not regularly used in the UK. Evening primrose oil is probably only effective for mastalgia, spironolactone for fluid retention and non-steroidal anti-inflammatory drugs for pain in the late luteal phase. Naltrexone has shown some benefit and requires further study.

Vitamin B6 - a co-factor in the synthesis of serotonin - has had a chequered history, particularly with the emergence of neurological damage at high dosage. A meta-analysis of a few placebo-controlled studies concluded that it is effective for PMS symptoms including depression at doses of up to $100 \mathrm{mg}$ daily (Wyatt et al, 1999).

Minerals such as magnesium, potassium and calcium have been beneficial in small open studies. The homeopathic remedy Vitex agnus castus fruit extract has proven helpful in a prospective randomised, placebo-controlled study (Schellenberg, 2001). Tibolone, a synthetic steroid, has been shown to improve symptoms and increase the serum $\beta$ endorphin concentration in a placebo-controlled cross-over study (Taskin et al, 1998).

\section{Ovulation suppression}

Danazol was one of the earliest drugs used to control premenstrual symptoms by suppression of ovulation. The side-effects, including masculinisation effects and raised lipids, have limited its use.

Gonadotropin-releasing hormone (GRH) agonists such as buserelin and leuprolide effectively suppress ovulation and are effective against a range of premenstrual symptoms. Freeman (1997) has shown that leuprolide is not effective for women who experience dysphoria throughout the menstrual cycle and have additional PMS symptoms, that is, the 'premenstrual exacerbation' group according to Steiner \& Wilkins's classification (see Box 3). Freeman postulates that these differential results suggest that premenstrual depression may have mechanisms different from those of other dysphoric mood disorders. The main disadvantage of GRH agonists is that they induce menopause. The early symptoms of this can be unpleasant but are abated by the addition of oestrogen and progesterone on a non-sequential basis. Sequential therapy can itself induce a PMS-like state. GRH agonists cannot be used in the longer term because of the risk of osteoporosis, but the beneficial effect can last for many cycles beyond the treatment phase.

Transdermal oestrogen patches can also suppress ovulation, however, unopposed oestrogen is not recommended unless hysterectomised. Progestins are usually added either orally or from a low-dose progestin-coated intra-uterine device. The advantage of oestrogen therapy with or without progestin is that it does not induce a menopausal state. Use of the oral contraceptive pill has been disappointing, including the use of continuous monophasic preparations, which minimise endocrine cyclicity. Ovariectomy is also curative, but such extreme irreversible surgery is rarely required.

Other hormonal treatments include the use of progesterone, however, several clinical trials have failed to show convincing efficacy.

As a psychiatrist, it can prove helpful to ask for the opinion of a gynaecologist for people with severe or persistent PMS, particularly when considering ovulation suppression. For detailed reviews and references, see Halbreight (1996) and Yonkers \& Brown (1996).

\section{Psychological models and lifestyle change in the management of PMS}

Psychologists have dispelled the myth that there is a universal premenstrual performance deficit in women. Work performance appears to be either unaffected or any deficit is either easily compensated for (Walker, 1997) or too subtle for standard testing. Women presenting with PMS, however, often do report more negative interpersonal experiences at work and doubt their competency in the premenstrual phase. Stereotypical views are held of how a woman feels and behaves premenstrually. On an individual basis, there may be an interaction between the acceptance of such stereotypes and subjective cognitive biases such as attributional errors, which then influences a woman's subjective experience of her mental and physical state premenstrually. Attributional biases may inflate the reporting of premenstrual symptoms, but do not negate the reality of a patient's distress.

Many studies of psychological interventions exist. These broadly subdivide into lifestyle changes such as dietary modifications, relaxation and exercise programmes, and specific therapeutic approaches such as support groups and cognitive-behavioural therapy (CBT). For detailed reviews and references relating to these areas, see Rivera-Tovar et al (1994) and Pearlstein (1996).

Suggested dietary modifications include reducing the intake of caffeine, alcohol, salt and unrefined 
sugar. Positive results have been noted in some studies, but these have largely been uncontrolled. Eating frequent small carbohydrate-rich snacks may increase the dietary availability of tryptophan, which in turn increases serotonin synthesis. Evidence is emerging for a beneficial effect on mood and craving of increasing carbohydrate intake premenstrually.

Relaxation training is a useful addition to behavioural treatment packages for PMS, but there is limited research evidence to support its use in isolation. Exercise has been more rigorously studied. Premenstrual complaints are lower among women who regularly engage in sporting activities. Introducing sedentary women to exercise has been shown to be beneficial for mood symptoms, fluid retention and breast tenderness when monitored prospectively for 6 months. Aerobics training has also been rated as beneficial, with high intensity being more effective than low intensity in a randomised controlled study of women with confirmed PMS. It is unclear if exercise-induced symptom improvement is a result of physiological or psychological changes.

Support groups tend to have varying areas of focus such as psychoeducation, problem-solving approaches or empathic listening. This heterogeneity renders it impossible to compare different group approaches, but women tend to find the group experience beneficial, particularly when talking to other women with similar problems.

\section{Cognitive-behavioural therapy}

Blake et al (1998) have described the application of a CBT model to the treatment of PMS, successfully borrowing established CBT techniques. They propose that women with PMS may be interpreting physiological changes in a negative way. The repeated expectation of a negative experience may intensify feelings of anxiety and depression, particularly where there are background psychosocial stressors. The physical changes expected may interfere with normal coping mechanisms, be viewed as unmanageable and further heighten apprehension and anxiety, leading to a sense of imminent loss of control. Circles of negative thinking and self-defeating behaviour maintain the maladaptive reaction to physiological changes. Of particular relevance are early life experiences of being criticised, which contribute to the setting of rigid standards for behaviour and performance and which make no allowance for mild fluctuations in well-being. When fluctuations occur they can be accompanied by maladaptive reactions. Using a CBT model in treatment involves attempting to find more adaptive ways of coping with premenstrual changes.

The use of CBT has been reviewed by Blake (1998) and Pearlstein (1996), with mixed results reported. Short-term interventions of four sessions or less have not proved helpful. Other CBT-based interventions have been shown to be superior to support groups, relaxation and dydrogesterone. As would be expected, CBT has been shown to be particularly effective in negative cognitions, self-blame and avoidance.

Interestingly Blake's study (1998) showed significant reductions in somatic symptoms such as breast tenderness and bloating, as recorded prospectively in diaries, in addition to psychological symptoms. They also highlight that the most robust changes seen were in specific PMS symptoms in comparison with depression or anxiety symptoms (having excluded patients suffering from significant psychiatric illness). There are no studies comparing CBT alone with the best available medication.

\section{Placebo effect}

No discussion of the treatment of PMS is complete without considering the placebo effect. Figures for placebo response vary and are usually around $30-40 \%$, but in one study of oestrogen with synthetic progestin the initial placebo response was $80 \%$ (Watson et al, 1989) and in another of surgically inserted oestrogen implants it was $94 \%$ (Magos et al, 1986). Little consideration is given in the literature as to what the placebo response in PMS research might represent. It is possible that the attention provided to subjects in a study may itself have a positive psychological effect - but it has not been shown why this should be particularly so for PMS. Cognitive models such as that of Blake (1998) may help explain this phenomenon. Women given treatment ' $X$ ' may have their negative expectations challenged, that is, the dysfunctional belief "I'll be premenstrual next week and I won't be able to cope" is no longer accepted unconditionally because the woman now has a reason to challenge it - treatment ' $X$ '. Expected negative changes in self-efficacy and reduced self-esteem are somewhat diluted, more adaptive coping mechanisms are accessed and the emotional and behavioural outcomes are less catastrophic, if not improved. This cognitive shift is then reflected in the daily self-ratings. The same process might explain the early success of some interventions that are not maintained over time. Short-term treatment studies may not adequately control for this initial effect. 


\section{Conclusions}

Our understanding of PMS, or the operationally defined PDD, has crept towards an acceptance of a bio-psychosocial model of aetiology and treatment.

Key aspects in the diagnosis and management of women presenting with premenstrual symptoms include detailed history-taking, prospective diary recording of symptoms and the exclusion of other significant medical and psychiatric disorders in order to allow a clear diagnosis to be made. Treatment strategies are driven by symptom severity but for most women will include both pharmacological and non-pharmacological approaches. SSRIs have consistently been shown to be of benefit and can be effective when used intermittently in the premenstruum. Ovulation suppression is particularly effective but is best carried out in liaison with a gynaecologist.

Non-pharmacological approaches vary in the degree to which they have been evaluated and remain a useful starting point for less severely affected individuals. CBT for severe PMS is acceptable to women and there is theoretical and preliminary research evidence to support its use in these cases. The effects of combining CBT with the use of SSRIs or ovulation suppressors has not been assessed by controlled trials but may offer a potentially rewarding approach for more severely affected women.

\section{References}

American Psychiatric Association (1980) Diagnostic and Statistical Manual of Mental Disorders (3rd edn, revised) (DSM-III-R). Washington DC: APA

- (1994) Diagnostic and Statistical Manual of Mental Disorders (4th edn) (DSM-IV). Washington DC: APA.

Blake, F., Salkovskis, P., Gath, D., et al (1998) Cognitive therapy for premenstrual syndrome: a controlled trial. Journal of Psychosomatic Research, 45, 307-318.

Budeiri, D. J., Li Wan Po, A. \& Dornan, J. C. (1994) Clinical trials of treatments of premenstrual syndromes: entry criteria and scales of measuring treatment outcomes. British Journal of Obstetrics and Gynaecology, 101 689-695.

Endicott, J., Halbreich, U., Schacht, S., et al (1981) Premenstrual changes and affective disorders. Psychosomatic Medicine, 43, 519-529.

Eriksson, E. (1999) Serotonin re-uptake inhibitors for the treatment of premenstrual dysphoria. International Clinical Psychopharmacology, 14(suppl. 2), S27-S33.

Frank, R. T. (1931) The hormonal causes of pre-menstrual tension. Archives of Neurological Psychiatry, 26, 1053-1057.

Freeman, E. W. (1995) Relationships between age and symptom severity among women seeking medical treatment for premenstrual symptoms. Psychological Medicine, 25, 309-315.
- (1997) Gonadotrophin-releasing hormone agonist in the treatment of premenstrual symptoms with and without ongoing dysphoria: a controlled study. Psychopharmacology Bulletin, 33, 303-309.

Gallant, S. J. Popiel, D. A., Hoffman, D. M., et al (1992) Using daily ratings to confirm premenstrual syndrome/ late luteal phase dysphoric disorder. Part II. What makes a "real" difference? Psychosomatic Medicine, 54, 167-181.

Greene, R. \& Dalton, K. (1953) The pre-menstrual syndrome. $B M I, 1,1007-1014$

Halbreight, U. (1996) Premenstrual syndromes. In Psychiatric Issues in Women (U. Halbreight), pp. 667-686. London: Baillière Tindall.

Magos, A. L., Brincat, M. \& Studd, J. W. (1986) Treatment of premenstrual syndrome with subcutaneous estradiol implants and cyclical oralnorethisterone; placebo controlled study. BMJ, 292, 1629-1633.

O'Dwyer, J., Friedman, T. \& Clifford, E. (1997) The relationship between menstruation and psychiatric admissions. Irish Journal of Psychological Medicine, 14, 4648 .

Pearlstein, T. B. (1996) Nonpharmacologic treatment of the premenstrual syndrome. Psychiatric Annals, 26, 590-594.

- \& Stone, A. B. (1998) Premenstrual syndrome. The Psychiatric Clinics of North America, 21, 577-590.

Rapkin, A. J. (1992) The role of serotonin in premenstrual syndrome. Clinical Obstetrics and Gynaecology, 35, 629-636.

Rivera-Tovar, A., Rhodes, R., Pearlstein, T. B., et al (1994) Treatment efficacy. In Premenstrual Dysphorias. Myths and Realities (eds. J. H. Gold \& S. K. Severino). Washington, DC: Americal Psychiatric Press.

Roca, C. A., Schmidt, P. J., Bolch, M., et al (1996) Implications of endocrine studies of premenstrual syndrome. Psychiatric Annuals, 26, 576-580.

Rubinow, D. R. (1992) The premenstrual syndrome. Journal of the American Medical Association, 268, 1908-1912.

— \& Schmidt, P. J. (1995) The treatment of premenstrual syndrome. Forward into the past. New England Journal of Medicine, 332, 1574-1575.

Sampson, G. A. (1988) Definition of premenstrual syndrome and related disorders. In Functional Disorders of the Menstrual Cycle (eds M. G. Brush \& E. M. Goudsmit). Chichester: John Wiley \& Sons Ltd.

Schellenberg, G. R. (2001) Treatment of the premenstrual syndrome with agnus castus fruit extract: prospective, randomised, placebo controlled study. BMJ, 322, 134138.

Schmidt, P. J., Nieman, L. K., Grover, G. N., et al (1991) Lack on effect of induced menses of symptoms on women with premenstrual syndrome. New England Journal of Medicine, 324, 1174-1179.

Steiner, M. \& Wilkins, A. (1996) Diagnosis and assessment of premenstrual dysphoria. Psychiatric Annuals, 26, $571-575$.

Taskin, O., Gokdeniz, R., Yalcinoglu, A., et al (1998) Placebocontrolled cross-over study of effects of tibolone on premenstrual symptoms and peripheral beta-endorphin concentrations in premenstrual syndrome. Human Reproduction, 13, 2402-2405.

Walker, A. E. (1997) The Menstrual Cycle, pp. 88-114. London: Routledge.

Watson, N. R., Studd, J. W. W., Savvas, M., et al (1989) Treatment of severe premenstrual syndrome with oestradiol patches and cyclical oral norethisterone. Lancet, ii, 730-732.

World Health Organization (1992) International Classification of Diseases (10th edn) (ICD-10). Geneva: WHO.

Wurtman, J. J. (1989) Effect of nutrient intake on premenstrual depression. American Journal of Obstetrics and Gynaecology, 161, 1228-1234.

Wyatt, K. M., Dimmock, P. W., Jones, P. W., et al (1999) Efficacy of vitamin B-6 in the treatment of premenstrual syndrome : systematic review. BMJ, 318, 1375-1381.

Yonkers, K. A. \& Brown, A. (1996) Pharmacological treatments for premenstrual dysphoric disorder. Psychiatric Annuals, 26, 586-589. 


\section{Multiple choice questions}

1. Typical symptoms of PMS include:
(a) dyspareunia
(b) pelvic discomfort
(c) irritability
(d) carbohydrate craving
(e) dysmenorrhoea.

2. PMS:

(a) is most common in younger women

(b) in between $3 \%$ and $8 \%$ of women is severe

(c) indicates abnormal cyclical ovarian function

(d) can be explained solely by expectation bias

(e) is linked to abnormalities in central serotonergic function.

3. Effective treatments in the management of severe PMS include:

(a) gonadotrophin-releasing hormone analogues

(b) triphasic oral contraceptives

(c) avoidance of caffeine

(d) oestradiol

(e) CBT.
4. Effective CBT for PMS requires:

(a) collaboration between the therapist and client

(b) a dismissal of irrational thoughts

(c) the identification of self-defeating patterns of behaviour

(d) a dismissal of early life experiences

(e) a maximum of five sessions.

5. SSRIs:

(a) can be used exclusively in the luteal phase

(b) have fewer side-effects when used in PMS alone

(c) cannot be combined with hormonal treatments

(d) usually inhibit ovulation

(e) are poorly tolerated in PMS.

\section{MCQ answers}

$\begin{array}{llllll}1 & 2 & 3 & 4 & 5\end{array}$

a $\mathbf{F}$ a $\mathrm{F}$ a $\mathrm{T}$

b $T$ b $T$ b F

\section{Finding the evidence:}

\section{A gateway to the literature in child and adolescent mental health (second edition)}

\section{Edited by Angela Scott, Mike Shaw and Carol Joughin}

There is an unprecedented demand for scientific evidence in child and adolescent mental health. Finding the Evidence is a unique resource that provides busy clinicians with rapid access to the best available secondary research evidence. This second edition provides an essential resource for clinicians and includes:

- A simple guide to searching for research evidence.

- A list of the best research evidence on a wide range of subjects of relevance to child and adolescent mental health professionals. This includes systematic reviews, meta-analyses, guidelines and practice parameters identified by searching bibliographic databases and an expert selection of classic \& cutting edge papers and key books in the area.

- Search strategies to allow clinicians to identify randomised controlled trials and systematic reviews.

- A range of critical appraisal tools.

New to the second edition are:

- A synopsis for all topic areas covering details of the aetiology, diagnosis and prevalence of the conditions.

- Expert commentary on the current research base and gap between research and practice.

- A significantly expanded and updated range of research.

- Key developments in the management of individual conditions.

- Important messages for the development of services.

October 2001, ISBN 1901242 684, Price $£ 18.00$

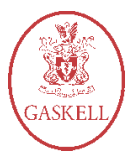

Available from Book Sales, The Royal College of Psychiatrists, 17 Belgrave Square, London, SW1X 8PG, UK. Tel: +44 (0)20 72352351 ext 146. Fax: +44 (0)20 72451231.

Website: www.rcpsych.ac.uk 


\title{
Editor, Advances in Psychiatric Treatment
}

The College is seeking a new Editor for Advances in Psychiatric Treatment (APT). The present Editor, Professor Andrew Sims, plans to stand down soon, having developed the journal over 7 years from a draft proposal to a thriving and highly regarded publication. Applications are invited from College members who feel they have the drive and vision to continue this work.

The post-holder will report to the College's Editor and to the APT Editorial Board. He or she will plan future content, commission articles, oversee the work of the Editorial Board and take responsibility for journal's content.

The post-holder should be committed to strengthening links with continuing professional development,while maintaining the independence of the journal; must be in touch with developments in evidence-based psychiatry; and will be aware of the needs of working psychiatrists across the range of sub-specialities. An understanding of the opportunities offered by on-line publishing would be an advantage.

It is anticipated that the successful candidate will devote at least one session per week to this role. The day-to-day administration and the production of the journal are handled by the College's Publications Department.

If you are interested in applying, please contact: Mr Dave Jago, Head of Publications, Royal College of Psychiatrists, 17 Belgrave Square, London SW1X 8PG (e-mail: djago@rcpsych.ac.uk) by 22 November 2001. Interviews will be held at the College in December.

\section{Child psychiatry and
child protection litigation}

\author{
By Julia Brophy
}

In the decade following the Children Act 1989 two issues have emerged as indisputable. First, care proceedings are a multi-disciplinary exercise and second, child and adolescent psychiatrists are key players providing the majority of expert evidence. This book is based upon a study that is the first to analyse and digest the views and practice of these clinicians after more than a decade's experience with the Children Act.

The text includes: - A thorough examination of the Children Act 1989.

- Detailed discussion of what the courts require of a clinical expert.

- Explores the controversial question of the 'added value' provided by experts in court.

- Discussion of how clinical opinion can be developed to improve family justice.

In a policy environment in which multi-agency approaches to child abuse and neglect are vital and where evidence-based practice increas ingly dictates clinical approaches, this book provides a new framework for child protection work and calls for wider debate about multi-disciplinary education and training for both lawyers and forensic experts. The book is central to future education and training initiatives for both new and established child and adolescent psychiatrists, as well as lawyers, magistrates, social workers and all other professionals involved with child protection litigation.

$$
\text { October 2001, ISBN } 1901242 \text { 668, Price } £ 20.00
$$

Available from Book Sales, The Royal College of Psychiatrists, 17 Belgrave Square, London, SW1X 8PG, UK

Tel: +44 (0)20 72352351 ext 146. Fax: +44 (0)20 72451231. 